\title{
ANALVSIS OF ELECTROMECHANICAL ACTUATOR BASED ON FEM - CIRCUIT SIMULATOR COUPLING
}

Abstract: Design and simulation of an electromechanical system is multidisciplinary. In this paper three different types of co-simulation of a mechatronic system are compared. The co-simulation is realized between two different programs (power electronic circuit simulator - control part and finite element analysis - magnetic and mechanic model part). The simulations were realized for an electromechanical actuator fuel injection system. The output waveforms for coil current, forces, inductances and position are compared. Finally the measured waveforms are presented too.

\section{Introduction}

Design and construction of modern types of electromechanical actuators is often verified by simulation. Analysis of a typical electromechanical system with power electronic converter consists of many aspects and is often multidisciplinary. Such a design can be realized only by different software packages. A classical electromechanical system consists of three different parts: electrical circuit (simulation of electrical circuit and control part), magnetic circuit (forces, co-energy, inductances) and mechanical part (torque, revolution). There is not a single simulation program which can handle such a system in a single way and consider all aspects at the same detailed level. Therefore, the design of an electromechanical system drive is mostly created step by step in different programs. The first step is usually building the magnetic circuit of a machine. Simulation results are often used in the next step, which is the simulation of electric circuit and control part of the machine. Simulation of mechanical loads and forces can be computed together with the first step in magnetic circuit simulation. However, the simulation of electric and magnetic circuit can not be decoupled because they interact with each other.

In this paper the simulation of a fuel injection system is investigated. There are two different parts in this system. Magnetic circuit and magnetic model specifications consist of forces, material properties and mechanical quantities such as movement, speed, shift and deformation. The electric circuit consists of a control circuit simulator together with the signal generator. To achieve an analysis of a complete system, two different programs are used. For this model type it is very suitable to use co-simulation, where two different programs are co-operating.

\section{Basic co-simulation principles}

In the first two cases of co-simulation it is possible to describe the program cooperation in the same way (Fig. 1). The difference is only in the obtained results. The dynamic power electronic model calculation is based on static finite element model calculation. From this the static values for inductance, magnetic force and coil current are obtained and used in the power electronic calculation. In the second case the difference is only for finite element model calculation where the power electronic output results were verified by the static loop calculation.

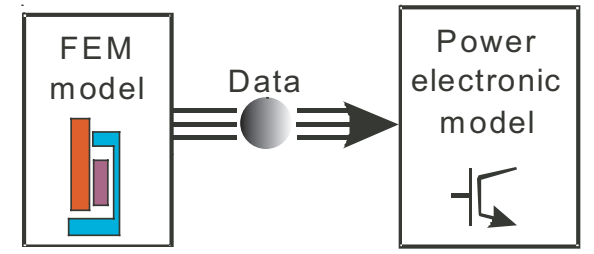

Fig. 1: Simple principle for data exchange at the first and second co-simulation

The computing like this one is accurate for a solenoid actuator description but from these first two co-simulation types there is no possibility to determine the transient behaviour. For this reason the third co-simulation possibility between the programs (Fig. 2) was realized. In this case it is necessary to realize the primary static computing in the finite element program too. After that the full dynamic co-simulation can start. This calculation is transient and during simulation the results are saved as data table.

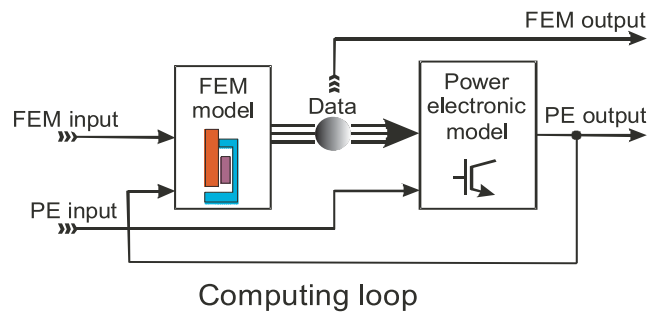

Fig. 2: Principle for full variable data exchange during co-simulation

\footnotetext{
* P. Bauer ${ }^{1}$, J. Sitár ${ }^{2}$

${ }^{1}$ Delft University of Technology/Power Electronic and Power Processing, Delft, The Netherlands, P.Bauer@tudelft.nl

${ }^{2}$ Alexander Dubcek University of Trencin, Faculty of Mechatronics, Trenčín, Slovakia
} 
The main question remains: on what level these programs must co-operate. Three different levels of data exchange will be defined as shown in Figs. 3, 4, and 5. These levels bring different kind of abstraction and simplification of the subsystems. Different kind of levels of the co-simulation will result in different output values. This paper will give an answer to the question, how the different level of co-simulation influences the results of the investigated system. The conclusions about the usefulness of these different levels of co-simulation are given. The best know simulation package for magnetic circuit analysis are Ansys, Femag, Femlab, Maxwell, Femm and there are many others. For simulation of electrical circuit the best know are Spice, Caspoc, Matlab/Simulink, Dynast and others. Some of these mentioned programs have a connection option with open interface, which can make the co-simulation. For example: Maxwell can be connected together with the Simplorer and Caspoc has a connection with Ansys and Matlab/Simulink Here co-simulation between Caspoc and Ansys will be created and the results are presented. Basic structures, analyses and mathematical models of solenoid are described in [8]. In literature the magnetic circuit model is presented together with control circuit.

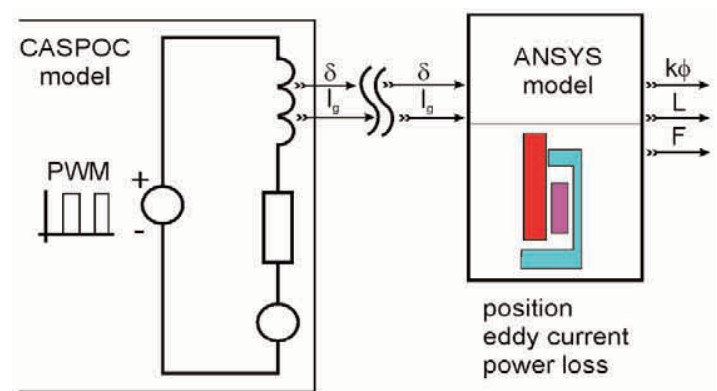

Fig. 3: Block circuit for one value co-simulation and data exchange

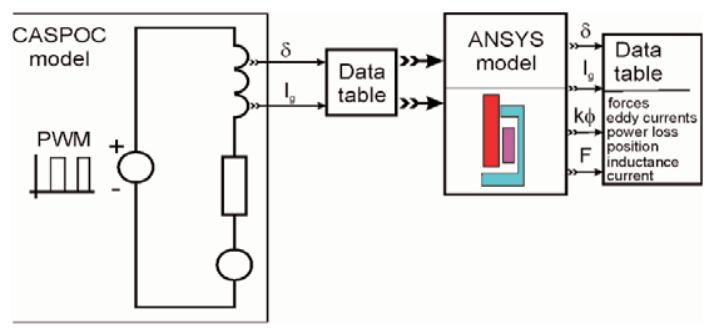

Fig. 4: Block diagram for table data exchange in co-simulation

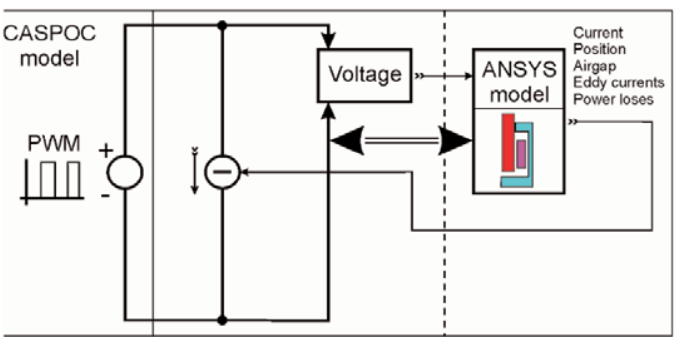

Fig. 5: Block scheme for full dynamic co-simulation (dynamic data exchange)

\section{Electromagnetic and mathematical model of solenoid}

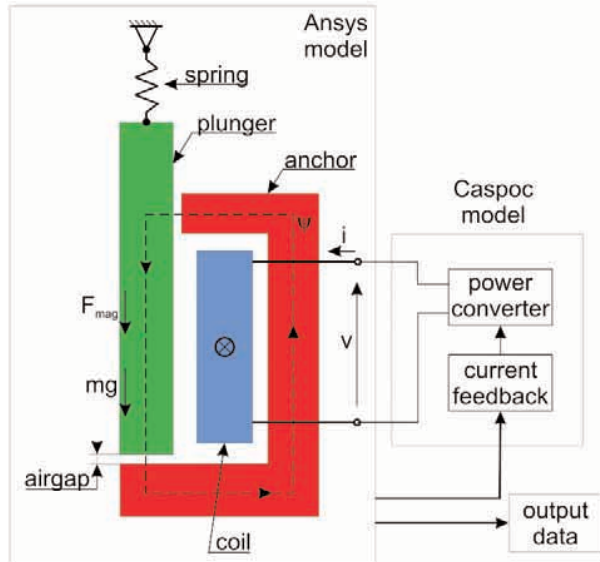

Fig. 6: Co-simulation model design

The principles of feedback current control strategy for solenoid are introduced and a simple electromagnetic actuator is developed. Here an analysis is carried out that calculates the inductance, flux densities, stored magnetic energy and co-energy, magnetic forces, current and power losses. Typical structures for the solenoid are described.

Fig. 6 shows the basic structure of the solenoid with a feedback current controller. Excitation of the windings produces magnetic forces for plunger movement. The plunger is free only in the vertical axis. The current $i$ produces a flux $\psi$. The flux path is shown by the dotted line in Fig. 6 and 7, and it crosses the air-gap in the vertical direction. The attractive force between the plunger and anchor is function of $i$ and is proportional to the square of $i$, if the material is not saturated. Under movement conditions, the generated attractive force is higher than the spring force. The spring force is a little bit higher than multiplied plunger weight $\mathrm{m}$ with gravity acceleration $g$. The force command is the sum of spring,
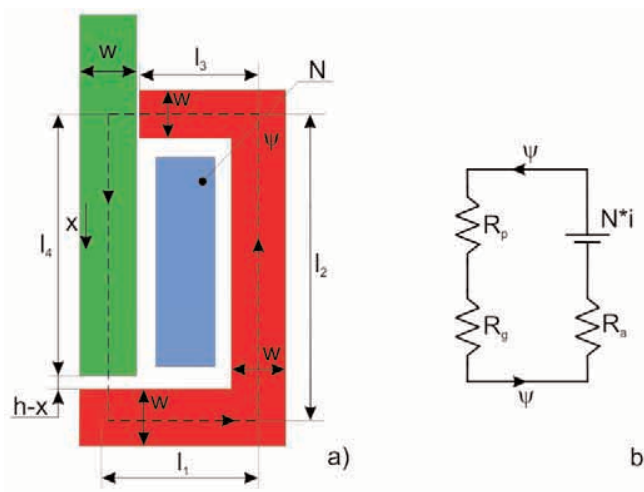

b)

Fig. 7: Solenoid basic dimensions and equivalent magnetic circuit a) and electric circuit $b$ ) 
gravity and attractive force commands. The spring force is in opposite direction to the gravity and attractive force commands.

The voltage $U$ excites a coil. Let us suppose that the number of turns in the windings is $N$ so that the magneto-motive force (MMF) $N i$ is produced. Since the permeability in ferromagnetic materials is high, the flux follows the path shown. The flux crosses the air - gap. Note that only one flux path is shown; however, the flux is distributed in the air - gap. The maximum flux density in the air - gap is determined by the coil excitations.

Figure 7 a) shows the model of the solenoid used for simulation. The anchor has the width $w$. The main flux path is indicated by the doted line. The length of the flux path in the model is defined by $l_{1}, l_{2}$ and $l_{3}$. The flux path length in the plunger is $l_{4}$. The winding in the coil has $N$ turns. The instantaneous current is $i$, so that the MMF is $N i$. The air-gap length is equal to $h$ at the maximal position. A coordinate position $x$ is defined in plunger position so that the air-gap length is $h-x$.

Reluctance of magnetic circuit is defined as:

$$
R=\frac{l_{f p}}{\mu_{m t} * S}
$$

Where:

$l_{f p} \quad$ - Flux path length $\left(l_{f p}=l_{1}+l_{2}+l_{3}+l_{4}\right)$

$\mu^{m t}$ - Permeability of the material

$S$ - Cross - section area of the flux path

Permeance is inverse function of magnetic reluctances:

$$
P_{a}=\frac{\mu_{m t} * S}{l_{f p}}
$$

Figure $7 \mathrm{~b}$ ) shows an "electrical" equivalent circuit of the solenoid magnetic circuit. In terms of MMF (voltage), flux (current), reluctances (resistance) and constant (dc) magnetic circuit can be treated in the same way as in the electric circuit. The main difference is that magnetic reluctance is an energy storage component rather than loss component. The "dc voltage" source $\mathrm{Ni}$ represents the MMF generated by the winding current. $R_{p}$ and $R_{a}$ are the magnetic reluctances in plunger and anchor respectively. $R_{g}$ represents the magnetic reluctance in the air - gap. These magnetic reluctances are written as:

$$
\begin{aligned}
& R_{a}=\frac{l_{1}+l_{2}+l_{3}}{\mu_{0} \mu_{r} S} \\
& R_{g}=\frac{h-x}{\mu_{0} S} \\
& R_{p}=\frac{l_{4}}{\mu_{0} \mu_{r} S}
\end{aligned}
$$

Where:

$\mu_{0}$ - is the permeability of free space $\left(\mu_{0}=4 \pi^{*} 10^{-7}\right)$

$\mu_{r}-$ is the relative permeability $\left(\mu_{m t}=\mu_{0} \mu_{r}\right)$
The flux $\psi$ is:

$$
\psi=\frac{N i}{2 R_{g}}=\frac{N i}{2} \frac{\mu_{0} S}{h-x}
$$

The flux linkage $\lambda_{1}$ of the coil is defined as a number of the turns $N$ multiplied by the flux passing through the coil:

$$
\lambda_{l}=\frac{N^{2} i}{2} \frac{\mu_{0} S}{h-x}
$$

Inductance is defined as flux linkage divided by the current value

$$
L=\frac{\lambda_{l}}{i}=\frac{N^{2}}{2} \frac{\mu_{0} S}{h-x}
$$

Stored magnetic energy and co-energy can be determined from the relation between flux linkage $\lambda$ and current $i$ in the magnetic circuit with ferromagnetic components such as the cores in figure 2 . The flux linkage is proportional only to low current values. At high current, the ferromagnetic cores become saturated, producing a nonlinear characteristic. Stored magnetic energy and coenergy is calculated in Ansys with Synergy macro. The stored magnetic energy $W_{m}$ in a magnetic system is obtained from:

$$
W_{m}=\int_{0}^{\lambda_{0}} i d \lambda
$$

In addition to the magnetic energy, we can introduce the magnetic co-energy $W_{m}^{\prime}$. The magnetic co-energy is defined as:

$$
W_{m}^{\prime}=\int_{0}^{\lambda_{0}} \lambda d i
$$

The independent variables in a magnetic solenoid system are normally the coil winding current and plunger position. If the system is moved by $\delta_{x}$ then it can be shown that the work done is equal to the change in co-energy of the system. Then, the electromagnetic force $F$ is given as the partial derivative of the magnetic co-energy:

$$
F=\frac{\partial W_{m}^{\prime}}{\partial x}
$$

If the magnetizing characteristic is linear (no saturation) then the magnetic stored energy is equal to magnetic co-energy. Assuming a linear system, where the self-inductance $L$ is constant and $L_{i}=\lambda$, the magnetic co-energy is derived as:

$$
W_{m}^{\prime}=\int_{0}^{i} L i d i=\frac{1}{2} L i^{2}
$$

Substituting (8) into (10) yields:

$$
F=\frac{\partial L}{\partial x} \frac{i^{2}}{2}=\frac{L_{0}}{h} \frac{i^{2}}{2}
$$

The next expression for magnetic force is based on flux density in the air-gap and is well-known as Maxwell stress equation where:

$$
F=\frac{B_{0}^{2}}{2 \mu_{0}} S
$$


$\mathrm{S}-$ is defined as the air-gap area and $B_{0}$ is nominal flux density. In this electromechanical system the nonlinear characteristic of the material properties are used.

\section{Control circuit model}

The control circuit model was created in power electronic and electrical circuit simulation software CASPOC. The full controlled four quadrant inverter is modeled here. Its parameters and values for output signal are described in TABLE 1 .

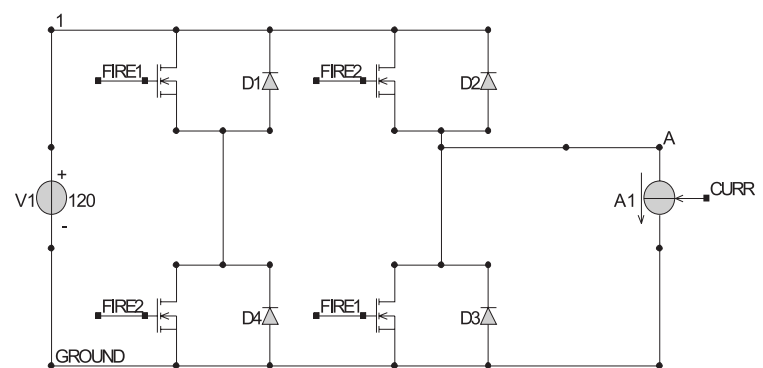

Fig. 8: Single phase full bridge controlled inverter implemented in power electronic circuit simulator.

Inverter parameters and output voltage waveform Table 1

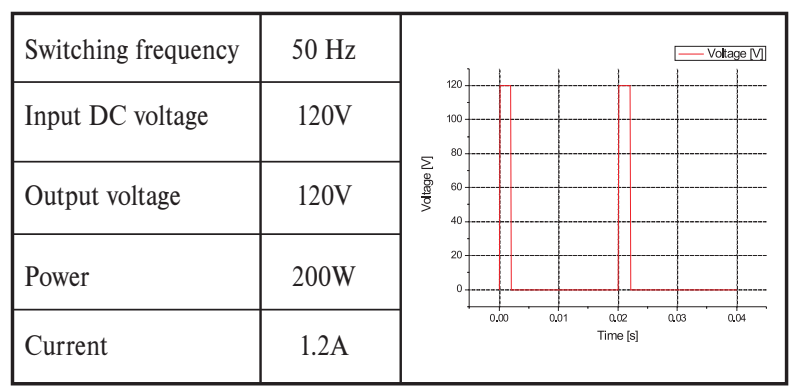

The electric circuit simulator also summarizes the finite element analysis results for value of inductance, power losses, eddy currents and forces and draws their waveforms in scopes. For co-sim- ulation the pre-defined function FROMLF (for receiving data from open interface) and TOLF (for sending data - to open interface) was used. The block scheme implemented in the power electronic circuit simulator of a single phase full controlled bridge inverter is shown in Fig. 8. This block diagram was prepared for co-simulation (there are co-simulation blocks and all the received data are visualized in the scope). All the received data was also used as an open interface in inverter simulation.

\section{Finite element model}

Magnetic circuit was created in finite element software ANSYS. There the mechanical and magnetic parts of solenoid were modeled together. Basic dimensions of model and material properties were described.

Basic input dimensions for finite element model

Table 2

\begin{tabular}{|l|c|}
\hline Model width & $15 \mathrm{~mm}$ \\
\hline Model height & $35 \mathrm{~mm}$ \\
\hline $\begin{array}{l}\text { Material types } \\
\text { (nonlinear material } \\
\text { properties - BH } \\
\text { curves) }\end{array}$ & $\begin{array}{l}\text { Air, Coil, } \\
\text { Two ferro- } \\
\text { magnetic } \\
\text { materials }\end{array}$ \\
\hline Plunger movement & $2.95 \mathrm{~mm}$ \\
\hline Spring constant & $1.065 \mathrm{e} 3$ \\
\hline Fill factor & 0.658 \\
\hline Number of turns & 1750 \\
\hline Spring damping & 0.05 \\
\hline
\end{tabular}

Outputs of this simulation are parameters for impedances, position, power losses, eddy currents and other requested results. These are, e.g., the waveforms for electromagnetic vector potential and magnetic flux density. The basic model dimensions are shown in TABLE 2.

In the finite element model a parameter value is computed and it is used via the open interface in the electric model simula-

\begin{tabular}{|l|l|l|}
\hline Type number & Realization & Description \\
\hline 1 st type & $\begin{array}{l}\text { One value data exchange without dynamic } \\
\text { co-simulation and without saturation effects } \\
\text { of electro-magnetic circuit }\end{array}$ & $\begin{array}{l}\text { Exchange of only one value for inductance, Force and Back-EMF from } \\
\text { the finite element model. No dynamic interaction between simulation } \\
\text { programs. }\end{array}$ \\
\hline 2nd type & $\begin{array}{l}\text { Table data ex-change without dynamic } \\
\text { co-simulation, but including saturation effects } \\
\text { of the electromagnetic circuit }\end{array}$ & $\begin{array}{l}\text { In this type the data table is used as input into simulation programs. These } \\
\text { tables include; for finite element model - values for position and coil current; } \\
\text { for power electronics - values for inductances, voltage back-EMF and force. }\end{array}$ \\
\hline 3rd type & $\begin{array}{l}\text { Full dynamic co-simulation considering } \\
\text { saturation effects of electromagnetic circuit } \\
\text { and transient eddy currents }\end{array}$ & $\begin{array}{l}\text { This simulation is based on full data exchange during simulation. All output } \\
\text { values of magnetic and electric circuit simulation are written in the graphs. } \\
\text { In this co-simulation finite element model and control power electronic } \\
\text { circuit simulator are mutually influenced during computation. }\end{array}$ \\
\hline
\end{tabular}


tion (Fig. 8). The electric control circuit computes the input voltage value for the finite element model and also exchanges them via the open interface. These values are used in the next step for each interconnected program. On this basic principle, a loop for co-simulation was created.

\section{Possibilities of co-simulation}

For computation, the FEM (finite element method magnetic circuit simulation) and power electronics circuit simulation software are used. In this paper we compare the results for the three different types of co-simulation (TABLE 3 and TABLE 4). The goal is to compare the difference between output values for coil current, inductance, force, air-gap for the mentioned three co-simulation possibilities. They are described below.

\section{VII. $1^{\text {st }}$ Co-simulation type}

The basis of this simulation is a simple calculation for one plunger position in the FEM software. For a constant coil current value, from FEM the inductance, force and back-EMF constant (induced voltage) can be obtained. The finite element model provides only one static value for these variables. For one plunger position in FEM the static magnetic vector potential or magnetic flux density can be presented.

The obtained results are used as inputs for power electronic circuit simulation program. The block diagram of this simulation type is shown in Fig.3. The plunger model is presented as output load on inverter DC link and is modeled by a mechanical system, where the plunger can move in one direction. This output load consists of a coil (FEM value of inductance), resistor (FEM value of resistance) and source of EMF (FEM value of induced voltage constant multiplied by plunger speed). From this simulation the waveforms for coil current, forces, and position can be drawn

\section{VIII. $2^{\text {nd }}$ Co-simulation type}

The block diagram of this simulation type is shown in Fig.4. For this co-simulation type the input and output values for inductance, back-EMF and force are in the data table form. The control circuit calculates an initial variable data table for different plunger position, voltage and coil current. This initial variable data table is converted into the finite element software script. This table is written cross the *DIM (defines an array parameter and its dimensions) commands for computation of macro ( ${ }^{*} \mathrm{DO}$ control for loop computation). In the finite element program these values of voltage and plunger position are read and the FEM program calculates the data table for inductance, back-EMF and force that are needed in its circuit simulation. This data table is written into a text file in column form. The next step is to create the waveform for these values in different software for comparison. Finite element output data can be used in the other control circuit calculation for variable value of inductance and coil current. In these first two cosimulation types only static computing was used in FEM analysis. These programs work separately all the time. These analyses are without dynamic co-simulation and only the second type consid-

Simulation types used in power electronic circuit simulator and finite element analysis

Table 4

\begin{tabular}{|c|c|c|}
\hline Type number & Power electronic simulation type & Finite element method simulation type \\
\hline 1st type & $\begin{array}{l}\text { Dynamic calculation - In the control circuit the } \\
\text { data table, which is used for finite element } \\
\text { model static computing is created. } \\
\text { After finishing the FEM analysis, the parameters } \\
\text { calculated in FEM are used in the transient } \\
\text { power electronic simulation. }\end{array}$ & $\begin{array}{l}\text { Static calculation - only one static value computation. For the next step, the } \\
\text { model parameters are adjusted based on control circuit data table manually } \\
\text { by user. }\end{array}$ \\
\hline 2nd type & $\begin{array}{l}\text { Dynamic calculation - In the control circuit the } \\
\text { data table, which is used for finite element } \\
\text { model in the static loop computing is created. } \\
\text { After finishing the FEM analysis, the table with } \\
\text { parameters from the FEM calculation is used in } \\
\text { the transient power electronics simulation. }\end{array}$ & $\begin{array}{l}\text { Static calculation together with data loop - based on results of control circuit } \\
\text { simulation. A data table is created to model the inputs for the *DIM areas. } \\
\text { These are used for changing the model parameters in the *DO loop. The } \\
\text { control circuit generates the data table which is used as input for finite } \\
\text { element model. Based on this table the finite element model is reconfigured } \\
\text { auto-matically and the results are calculated for each position listed in the } \\
\text { table. }\end{array}$ \\
\hline 3rd type & $\begin{array}{l}\text { Dynamic calculation - The Power electronic } \\
\text { simulator starts the co-simulation. In the first } \\
\text { step it computes and sends data for the driving } \\
\text { voltage. This data is sent to the FEM program } \\
\text { and the simulator waits for the next step. The } \\
\text { next step is realized after recalculation of the } \\
\text { magnetic circuit in FEM. The whole process is } \\
\text { repeated in a loop. After reaching the final } \\
\text { simulation end time, the co-simulation process } \\
\text { is terminated }\end{array}$ & $\begin{array}{l}\text { Dynamic calculation - in this case the full transients in the magnetic } \\
\text { circuit are modeled. The forward movement of the plunger is caused by } \\
\text { electro-magnetic force. The backwards movement is caused by a mechanical } \\
\text { spring (in the FEM model realized by a spring constant and spring damping) } \\
\text { The input value for the driving voltage is obtained from the power } \\
\text { electronic circuit simulation. The considered simulation results are values } \\
\text { for magnetic vector potential, magnetic intensity, inductance, forces, coil } \\
\text { current, speed and back-EMF. }\end{array}$ \\
\hline
\end{tabular}


ers saturation of the electromagnetic circuit. Eddy currents are not considered in the first and second type of co-simulation.

\section{IX. $3^{\text {rd }}$ Co-simulation type}

This co-simulation is based on full data exchange during simulation. The block diagram of this simulation type is shown in Fig. 5. All the data is exchanged between both programs at each time step in the simulation. The Power electronic simulation software continuously reads this data and in the same time computes the next voltage and position value for FEM software. The results are drawn into the oscilloscopes.

The interconnection between programs is realized inside power electronic and FEM software. In the power electronic software are the co-simulation blocks for data reading and writing (TOLF and FROMFL). The program creates for this purpose two different data sets. The first set for reading FEM results data and the second for writing the output voltage value for finite element software. The name of these datasets, co-simulation time and number of sub-steps can be set in power electronic software. In FEM analysis these data sets are used, where the first data set is used for data writing and second data set for input voltage reading. In this case the co-simulation includes the saturation of magnetic circuit and influence of eddy currents. During the saturation of magnetic circuit there are no changes for the material properties in this simulation set up. These properties are assigned with the assistance of $\mathrm{BH}$ curve. There are changes only in current values and inductance.

After finishing the co-simulation the waveforms for all the computed variable values are shown in graphs (Fig. 9 and 10). Transient FEM results are given as animations, variable data table and nonlinear model characteristics are given for magnetic vector potential and magnetic flux density. Some of the results are shown in the next section.

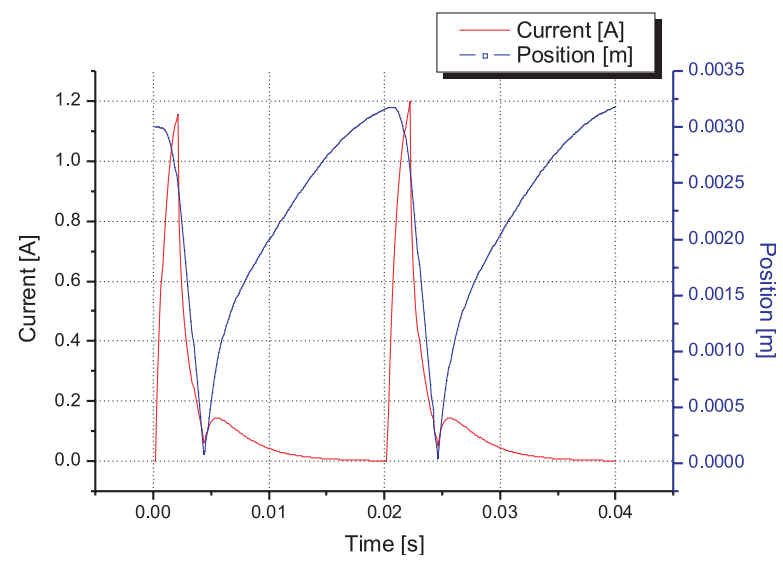

Fig. 9: Dynamic co-simulation results for air-gap and current waveforms (full dynamic co-simulation).

\section{Comparison of the results}

Different criterions for comparison of these three co-simulation methods are used. The first criterion is for time and hardware requirements. The first method uses not many system requirements, but if we need more results for different plunger position the user must change parameters before the next computation manually. The second method uses a variable data table. The computation is realized for many positions in one step, but not in the real interaction between programs. Control circuit computation is not time consuming, but the finite element calculation takes a long time and is dependent on the variable data table.

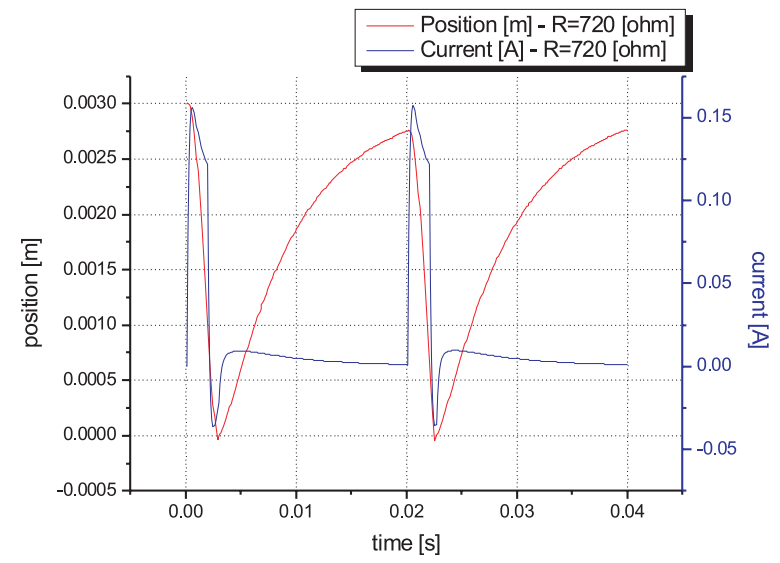

Fig. 10: Power electronic simulator results for air-gap and current (bases for this calculation is variable data table which was computed in static finite element analysis).

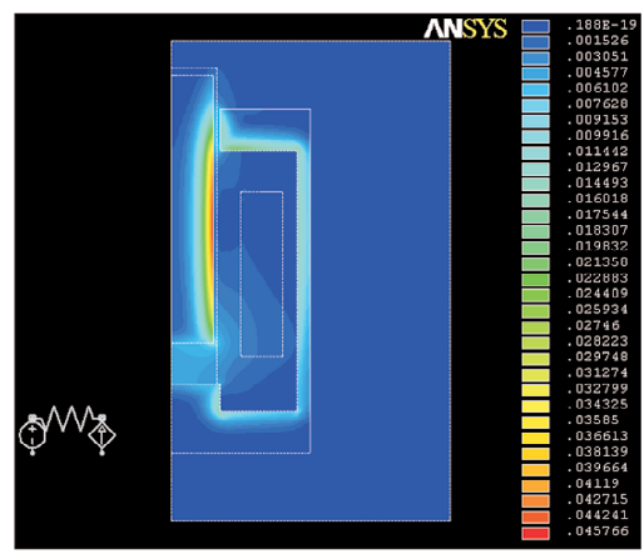

Fig. 11: Magnetic flux density at the maximum air gap $(l g=0.3)$.

The last co-simulation method is comparable with the second method with respect to the computing time (electric and magnetic model needed the same time for computing), but the results are different due to simplification made in the first two models (Figs. 3 and 4). 


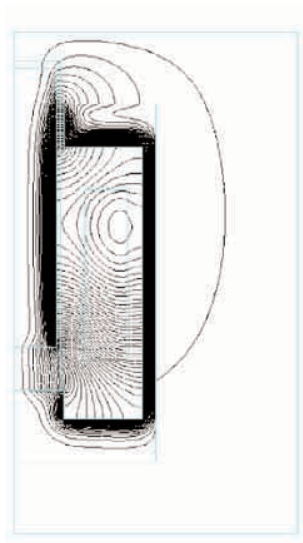

NNSYS

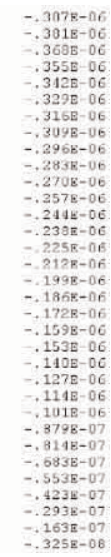

Fig. 12: Magnetic vector potential for maximum air gap $(l g=0.3)$.

The next criterion is the comparison of the acquired results. We can compare values of inductance, coil current, plunger position and forces. Values of these variables can be written in one graph. The output waveforms for current and position are shown in Figs. 9, 10, 13 and 14.

In Fig. 9 the full dynamic co-simulation results are presented (full cooperation between FEM analysis and power electronic simulation). The coil current (red) and plunger position (blue) are compared there. After starting the co-simulation, the coil current increases as long as there is a driving voltage. At the same time the plunger starts its movement to the zero position. After removing the driving voltage, the current decreases as long as the plunger position is zero. After reaching the zero position and sufficient current drop the backward movement is started. (Spring force is higher than electromagnetic force). Continuously, there is a small peak in the current waveform. It's the effect of induced voltage (back-EMF) and eddy currents. After this state the current is progressively decreasing to zero and air-gap is increasing to maximal position. In Fig. 10 are the same waveforms (red - plunger position, blue - coil current) for 2nd type of co-simulation.

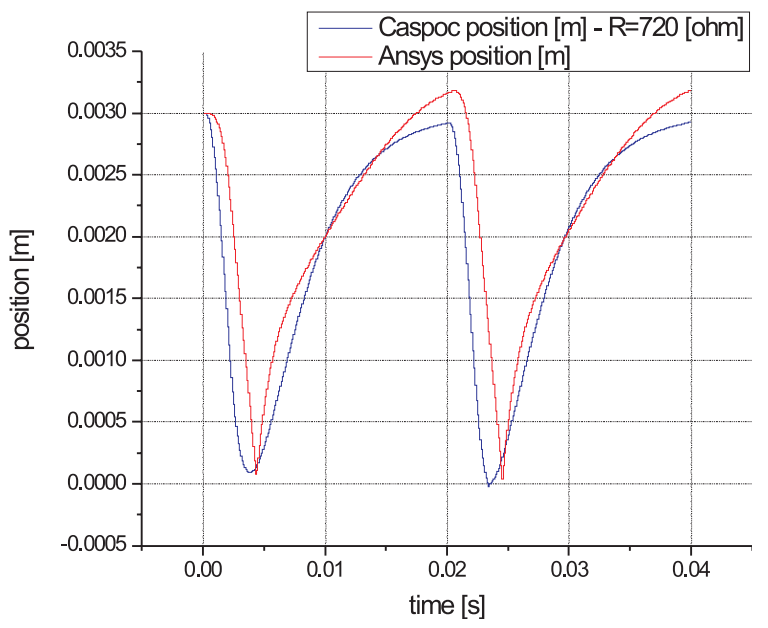

Fig. 13: Comparison of air-gap waveforms for two periods.
Figs. 11, 12, 15 and 16 show value for magnetic vector potential and magnetic flux densities during co-simulation for the maximum and minimum values of air-gap. The results of co-simulation are values for storage magnetic energy and co-energy, inductance, power losses, eddy current and coil current. These results were summarized during co-simulation in power electronic software. Their waveforms are presented in the figures below.

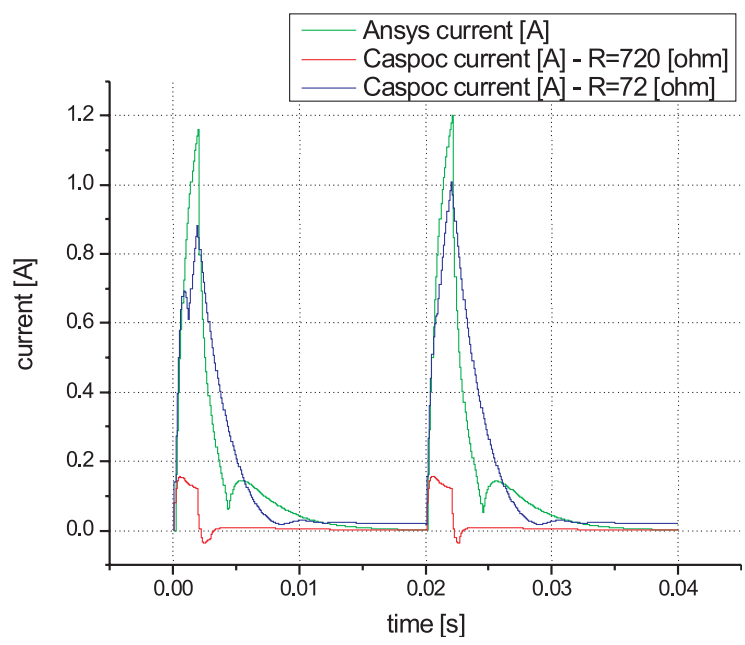

Fig. 14: Comparison of current waveforms for two periods.

In Figs. 13 and 14 is comparison between 2nd and 3rd co-simulation type in position and current. Fig. 13 shows the confrontation between plunger positions (noted as Ansys position - 3rd co-simulation type, noted as Caspoc position - 2nd co-simulation type). The basic difference is in velocity of air-gap reduction and in backward movement; where the third co-simulation result is above the maximum air-gap and the results from second co-simulation are bellow the maximum air-gap. In Fig. 14 the comparison between currents waveforms are presented (Ansys current - 3rd co-simulation type, Caspoc current - 2nd co-simulation type with different model resistance).

The current increases until the input voltage is connected. After removing the driving voltage the current value decreases until the plunger position is equal to zero. When the plunger starts the backwards movement, the current rises a bit. After this situation, the current decreases to zero continuously.

\section{Experimental validation}

In measurement the solenoid current waveforms for different size of duty cycle were obtained (Fig. 19). In these current waveforms it is possible to see the effect of the local current minimum. It is the result of mechanical movement of an inductor through a magnetic field. This phenomenon has very important diagnostic implications. Usually the circuit will have about 20 percent to 40 percent more current than is necessary to pull in the coil so it is possible to see about $2 / 3$ to $3 / 4$ of the way up the ramp. The size 
and distinction are directly related to the amount of movement. The actual mechanical movement in the fuel injector takes place in about 0,5 millisecond.

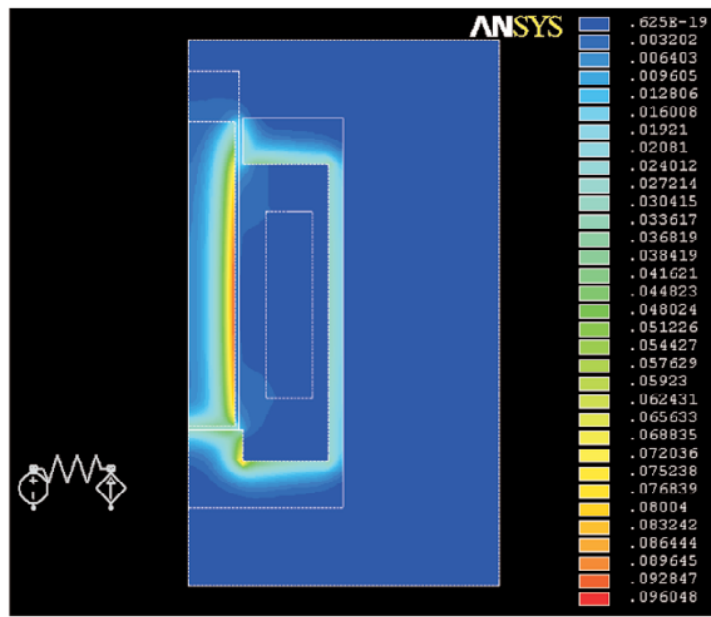

Fig. 15. Magnetic flux density at the minimum air gap $(l g=0)$.
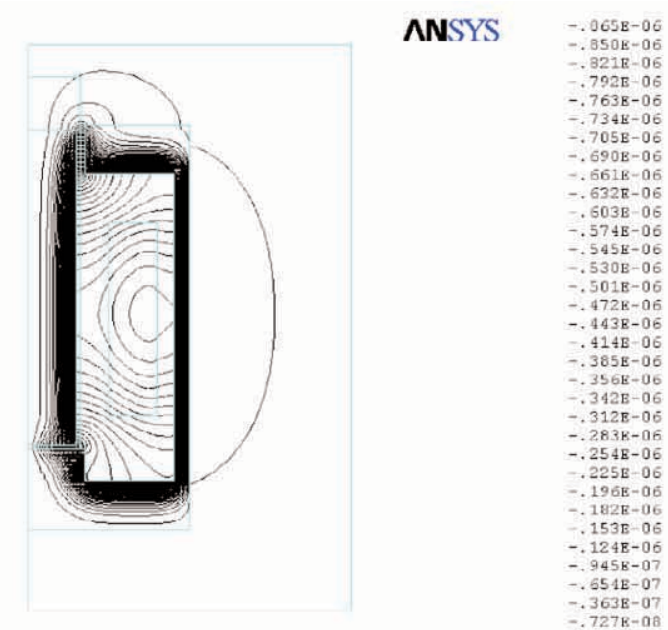

Fig. 16. Magnetic vector potential and magnetic flux density at the minimum air gap $(\lg =0)$.

To better illustrate this phenomenon, it is possible to use a simple relay. A relay coil has a multitude of windings that electrons must pass through before completing a circuit. Magnetic lines of force are created around the conductor. The lines cut across the adjacent windings causing their electrons to move in opposition to the main flow. This is called back-EMF or Counter Electromotive Force (CEMF).

When an inductor is placed in the core of a coil, the magnetic lines of force are attracted to the inductor; as current flows, they concentrate on it causing more CEMF and slow current build-up in the coil. Then as the magnetic field builds enough strength to

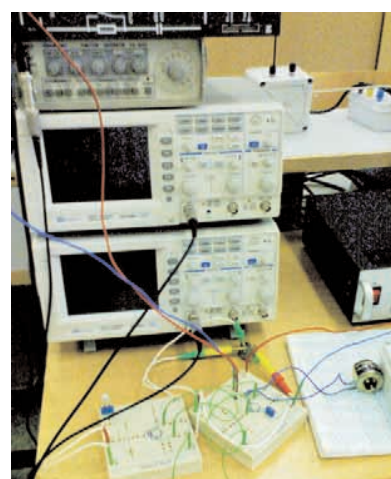

Fig. 17. Test bench

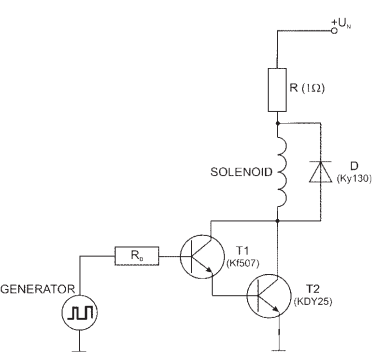

Fig. 18. Basic circuit for solenoid current measurement pull in the inductor, a fluctuation occurs causing the current flow to lessen momentarily as the inductor moves through its travel to a stop. After that it is possible to see the decreasing of current which is in literature presented as a Gull Effect. After saturation of the coil, the current will gradually reach the peak.

In Fig. 18 a simple electronic circuit for solenoid current measurement is presented. The current is calculated with Ohm's law from measured voltage on a precise resistor $\mathrm{R}$. The solenoid voltage was $90 \mathrm{~V}$ and maximum current value was $136 \mathrm{~mA}$.

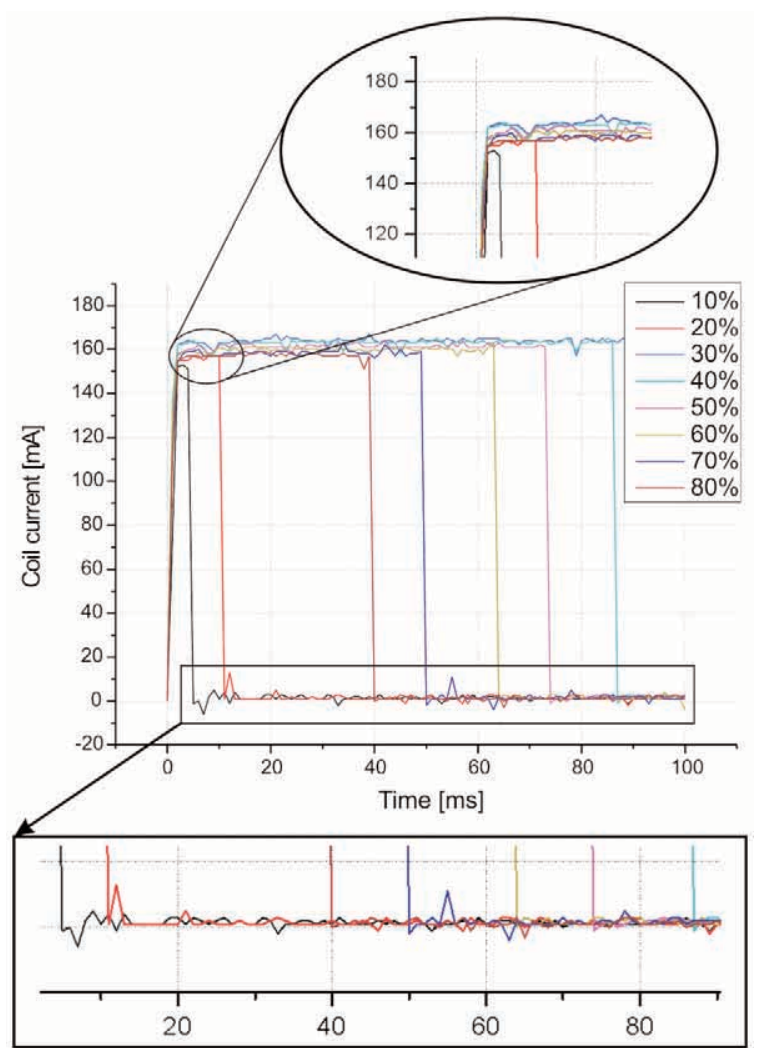

Fig. 19. Comparison of current waveforms for one period and different duty cycle 
Fig. 19 shows the solenoid current reading consistently throughout its range up to 80 percent duty cycle along with the voltage signals. At 100 percent duty cycle there would be a $100 \mathrm{~ms}$ pulse. In presented waveforms it is possible to see the gull effect in the beginning. After finish of voltage pulse the current is decreasing. Near the zero value the current increases a little bit and after that it drops to zero.

\section{Conclusions}

A full dynamic co-simulation for an electromechanical system in power electronic circuit simulator and finite element analysis is presented in this paper. In the FEM program ANSYS the magnetic part of solenoid model was computed. In power electronic simulator CASPOC the control circuit values were calculated. The two programs exchange data during the co-simulation and exact values and dynamical simulation are achieved. Both subsystems (electrical and electromechanical) are simulated in full detail. Such a co-simulation allows achieving more precise simulation results and, which is most important; it can include the transient behavior in the magnetic material such as eddy currents. Finally, the output values for current and position were compared and the measurement of a real solenoid current waveform was presented.

\section{References}

[1] ANSYS 9.0, www.ansys.com

[2] Caspoc 2005, www.caspoc.com

[3] CHIBA, A., FUKAO, T., ICHIKAVA, O., OSHIMA, M., DORRELL, G. D.: Magnetic Bearings and Bearingless Drives, an print of Elsevier, ISBN 07506557278

[4] REEVE, J. M., POLLOCK, C.: Dynamic simulation model for two-phase mutually coupled reluctance machines, Industry Applications Conference, 2001. Thirty-Sixth IAS Annual Meeting. Conference Record of the 2001 IEEE,Volume 1, 30 Sept.-4 Oct. 2001, pp. 40-47, vol. 1, Digital Object Identifier 10.1109/IAS.2001.955390

[5] SUN, H.; LU, P.; ZHANG, P.; CHEN, H.: Dynamic analysis of AT-cut quartz resonators with ANSYS, Sensors, 2004. Proceedings of IEEE, 24-27 Oct. 2004, pp. 95-98, vol. 1, Digital Object Identifier 10.1109/ICSENS.2004.1426108

[6] SUN, H.; LU, P.; ZHANG, P.; CHEN, H.: 3D ANSYS quench simulation of cosine theta Nb/sub 3/Sn high field dipole magnets, Applied Superconductivity, IEEE Transactions on, 2/2004, pp. 291-294, Digital Object Identifier 10.1109/TASC.2004.829088

[7] ZHIQIANG MENG; ZHONGDONG WANG.: The analysis of mechanical strength of HV winding using finite element method. Part I Calculation of electromagnetic forces, Universities Power Engineering Conference, 2004. UPEC 2004. 39th International,.Vol. 1, 6-8 Sept. 2004, pp. 170-174

[8] SITAR, J., BAUER, P.: Detailed analysis of Electronics for Solenoid Actuator with FEM and Circuit Simulator, Power Electronic Intelligent Motion Power Quality, May 30 - June 1, 2006, Nurenberg, Germany, pp. 124-135.

[9] MAGA, D., HARTANSKY, R.: Numerical solution, 1. issue, Brno, University of defense, 2006, p. 174 - ISBN 80-7231-130-1. 